\title{
Standard cooperating procedures
}

\section{Community review of proposed standards is a good strategy to broaden consensus on ways to conduct principled, ethical and efficient research. We are pleased to welcome new partners for our Nature Precedings Data Standards initiative and suggest other standards that could be usefully presented as citable preprints.}

$T_{\text {hiser }}^{\text {hes }}$ his issue publishes a Correspondence and a Perspective, both developed by community peer review on our preprint server dedicated to Data Standards (http://precedings.nature.com/tags/ standards\%20paper). The Perspective by Matthew Freedman et al. ( $\mathrm{p}$ 513) proposes principles for functional characterization of loci identified by genome-wide association studies (GWAS). It was initially drafted by researchers concerned with standards for postGWAS analysis of cancer risk loci and was revised after numerous contributions and criticisms accrued over a period of months of community review. The revisions and many of the comments remain fully public on the preprint site, and we were able to publish the revised version without further need for supervised peer review. Although the recommended strategies have been developed in depth in order to make genomic results useful primarily to other cancer researchers, they are general enough to form the basis for functional analysis of loci conferring risk of other diseases.

The Correspondence by Anne Cambon-Thomsen et al. ( $\mathrm{p} 503$ ) proposes data citation with associated metrics for biobanks, databases and bioinformatics tools to promote data sharing. If widely implemented, this practice would have important implications for efficient resource allocation and would credit those initiating and supporting research as well as those writing up its results. The granularity of credit remains to be elaborated, but it will certainly recognize individuals and their roles via the Open Researcher and Contributor ID (ORCID) researcher identifier. If individual research subjects wish to track their own contribution to research, careful attention to identity escrow will be needed. For example, credit for research on anonymised DNA samples donated by individuals with a particular disease might accrue to their diseasespecific support group. Aggregated reputation credit of this kind may be enough to ensure future participation, support and funding without opening discussion on the impossibility of guaranteeing both individual credit and continued individual anonymity.

Our community approach extends not only to our own site but to those of other publishers, funders, informaticians and research consortia. In this respect, we are pleased to work with the Biosharing project (http://otter.oerc.ox.ac.uk/biosharing/).
The Data Standards preprint server at Nature Precedings makes funder policies, data plans and standards documents citable for use and comment. Biosharing complements this by indexing and presenting them along with other related initiatives. By working together to maximize the visibility and inter-operability of policies, plans and standards, we will make them easier to develop and submit and improve their usefulness to researchers.

Ethics committees and institutional review boards are another group that run the risk of developing locally appropriate but globally incompatible standards. This may not matter for the lifetime of one study, but it is rare that a single institution's study provides publishable results without raising further research questions. Notably, the consortium research published in the last few years by the journal showcases the efficiency of combining studies, as meta-analyses and combined analyses of datasets from many sites have the power to make discoveries that local datasets are too small to support. In this research environment, local informed consent agreements that unnecessarily restrict reuse of data can seriously impede research without necessarily providing better protection for research subjects.

So, there is a need to record and harmonize ethics agreements for optimal protection and research compatibility. In the United States, both the Food and Drug Administration (http://www.fda.gov/) and the Office for Human Research Protections (http://www.hhs.gov/ohrp/policy/ictips.html) provide instructions on both compliance with legislation and best practice. Because much of the research in genomics and epidemiology requires the use of data collected internationally, there is also a need to share detailed consents more widely. Voluntary public sharing of the detailed consents developed and applied at each institution would provide welcome feedback as well as examples of best practice that can be unified with those applied in other jurisdictions. Open sharing of the detailed consents has the additional benefit of limiting local divergence (preventing the reinventing of wheels of different sizes) in accordance with the universal principles for the protection of research participants. 